\title{
Tidal Stream Turbine Power Capping in a Head-Driven Tidal Channel
}

\author{
C. R. Vogel ${ }^{\mathrm{a}, *}$, R. H. J. Willden ${ }^{\mathrm{a}}$, G. T. Houlsby ${ }^{\mathrm{a}}$ \\ ${ }^{a}$ Department of Engineering Science, University of Oxford, OX1 3PJ, United Kingdom
}

\begin{abstract}
An array of ten $1 \mathrm{MW}$ turbines, with a one diameter inter-turbine spacing, is simulated in a hypothetical tidal channel using the depth-averaged flow solver TELEMAC-2D. It has been shown for idealised turbines that mean power extraction from tidal channels can be enhanced by tuning turbine resistance for a given channel. Blade element momentum theory is used to analyse turbine control strategies such as power capping, which can allow turbine operation to better match the dynamics of the underlying tidal resource. It is shown that choosing control strategies that limit array thrust helps sustain higher channel flow speeds, and hence can lead higher mean power production than other strategies. This has an impact on tidal farm size, where mean turbine power reduces as the number of turbines increases, due to the reducing flow rate in the channel. Adding turbines beyond the channel and turbine specific optimal chokes the flow, reducing overall farm power. To limit environmental impact it may be desirable to constrain the maximum flow-rate reduction. Under a $10 \%$ flow-rate reduction constraint, the lower thrust per turbine under pitch-to-feather control allows significantly more turbines to be installed and greater farm power than either pitch-to-stall or no power capping strategy.
\end{abstract}

Keywords: Tidal stream power, power capping, tidal turbine array, depth-averaged flow

The tidal stream resource presents an attractive source of clean and predictable, albeit periodic, renewable energy. Despite the multiplicity of concepts for harnessing tidal stream energy, it is generally accepted that a large number of such devices will be required to deliver a significant amount of power to national electricity grids [1]. The most attractive sites for tidal stream energy extraction are in regions where the energy transmitted from the deep oceans is concentrated by local tidal dynamics resulting in high flow speeds due to resonances established with the continental shelf, tidal constituents, and local bathymetric features which act to focus the energy [2]. The dominant tidal constituents at a site arise as a consequence of these interactions between the continental shelf and deep oceans, and hence differ around the world. Quarter wavelength resonance is established with the M2 (lunar semi-diurnal) and S2 (solar semi-diurnal) tidal constituents in the Bay of Fundy, Canada, generating a tidal range of over 10 metres, whereas the K1 and O1 lunar diurnal tidal constituents are resonant in the Gulf of Mexico [3].

Early attempts to develop methodologies to assess the large-scale tidal stream energy resource, for example Black and Veatch [4] around the UK, were based on observations of the undisturbed kinetic flux at sites of interest, similar to the analysis of the wind energy resource. Subsequent work has however revealed that there is a complex interaction between tidal energy extraction and the local tidal dynamics, which in turn affects the available tidal stream resource. Garrett and Cummins [5] developed an analytic model that approximated tidal turbines as a region of enhanced resistance to flow in a tidal channel between two large bodies of water, and showed that a limit to the available power in a tidal channel could be expressed as a function of the bed friction and tidal amplitude in the channel. Maximal power extraction was shown to require a significant reduction in the channel flow rate. The underlying channel model has since been extended in various ways, including modelling turbine bypass flows [6], analogy to electrical circuits [7], and considering different tidal

\footnotetext{
* Corresponding author

Email address: christopher.vogel@eng.ox.ac.uk (C. R. Vogel)
} 
basin dynamics [8]. A key outcome of such studies is that the power potential of a tidal channel depends not only on the undisturbed kinetic flux, but also on the driving tidal amplitude and on the natural drag and geometry of the channel. The flow through channels with low levels of natural drag can be significantly affected by even relatively small tidal farm deployments, whereas much larger tidal farms can be deployed in channels with high natural drag for the same reduction in flow rate. It has been demonstrated that large tidal farms can have a substantial impact on the phase of the flow through the channel [9], and that theoretical limits to available power could be improved by varying the turbine resistance in the channel throughout the tidal cycle [10].

The interaction between energy extraction and the underlying tidal resource, and specifically the balance between the natural drag in the channel and the resistance presented by the turbine farm, has also been demonstrated through more detailed computational studies (e.g. [11, 12, 13, 14]). The impacts of tidal farms are site-specific, as the natural tidal dynamics also depends on factors such as the presence of tidal flats, channel bathymetry, and turbine placement.

Although the analytical and numerical models discussed above allow the interactions between local tidal dynamics and energy extraction to be investigated, the simplified turbine representations used typically do not reflect the performance characteristics of real turbines. In particular, parameterisation of the actuator disk turbine emulator performance in terms of the wake induction factor does not provide a realistic description of turbine operation and can lead to infeasibly high levels of thrust being inferred. Device-scale simulations (e.g. $[15,16])$ provide more complete descriptions of device performance, but are generally solved with a constant inflow boundary condition and therefore do not simulate the reduction in flow rate in a headdriven tidal channel due to the additional resistance of the turbines. Implementation of more realistic turbine models within basin-scale tidal resource assessments is required to better understand the effect of tidal energy extraction on the tidal resource.

Enhancing the economic and technical feasibility of energy extraction requires that turbine capacity is matched to the magnitude and temporal variability of the available resource. Fluctuations in the tidal resource occur over a range of time scales, from several hours, to days, and even years. In general, device cost scales with the maximum power of the device and therefore rated flow speed, so that the capacity of the turbine will be selected to best match the device cost to the revenue generated and may result in only a fraction of the peak available resource being harnessed. Control strategies are required if the relationship between device performance and flow speed changes in order to maintain a specified rated power, which will in turn have an effect on the interaction between energy extraction and the local tidal dynamics. A common method used by wind turbines to maintain rated power is altering turbine blade pitch to change the angle of attack of the blades. Two main strategies exist: increasing the blade pitch in order to reduce the angle of attack (hereafter referred to as pitch-to-feather); or to decrease blade pitch to stall the blades (hereafter referred to as pitch-to-stall). Whilst pitch-to-feather control is widely and successfully used in the context of wind turbines, the levels of turbine thrust required at some points through the tidal cycle to maximise average power implied by idealised analyses such as [9] and [10] may necessitate alternative strategies such as pitch-to-stall control.

This paper investigates the effect of power capping on the performance of a tidal turbine fence in a tidally oscillating channel. Utilising turbine performance characteristics in contrast to an actuator disk in the analysis has the advantage of ensuring that the impact of energy extraction on the tidal dynamics reflects how turbines might be operated. It should be noted, however, that this means that the results presented herein do not reflect the theoretical limit to the power that could be extracted from a tidally oscillating channel. The thrust and power profile for a turbine have been derived using blockage-corrected blade element momentum theory for pitch-to-stall and pitch-to-feather cases [17]. The thrust and power profile are used to parameterise an array of tidal turbines that are deployed in a depth-averaged shallow water model of an idealised tidal channel located between two large ocean basins. 
a)

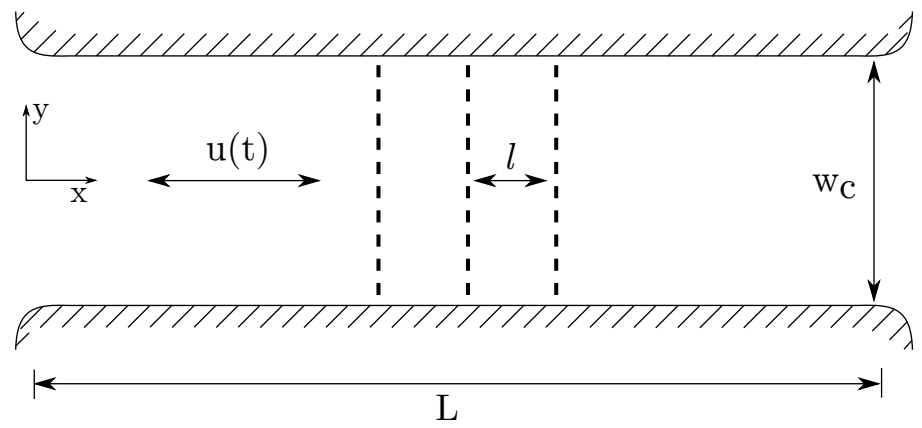

b)

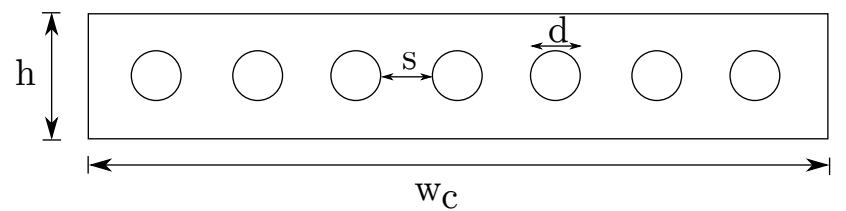

Figure 1: a) Schematic of the tidal channel of length $L$, uniform depth $h$, and constant width $w_{c}$, which lies between two large oceanic basins to the east and west of the channel. The flow speed in the channel is a function of time, the variation in head at either end of the channel, and the resistance to flow in the channel. Multiple cross-stream rows of turbines, with inter-row spacing $l$, may be deployed in the channel. A cross-sectional diagram of the channel is shown in b). Turbines, of diameter $d$, are uniformly arrayed across the channel cross-section within each row, with an inter-turbine spacing of $s$.

\section{Computational model}

The power extracted by a tidal fence with turbine power capping in a hypothetical head-driven tidal channel between two large bodies of water, such as that of a small channel between two islands e.g. Lashy Sound or South Ronaldsay Channel, has been simulated in TELEMAC-2D. TELEMAC-2D is a widely used open-source hydrodynamic modelling suite that uses a finite element approach to simulate depth-averaged free-surface flows, assuming hydrostatic pressure variation. Figure 1 illustrates the channel, in the central portion of the computational domain, in which turbines were deployed. The channel is $4000 \mathrm{~m}$ long and $400 \mathrm{~m}$ wide, expanding smoothly into large basins of radius $12000 \mathrm{~m}$ at either end with rounded inlets and outlets of radius $2000 \mathrm{~m}$. The mean depth of the channel and surrounding basins was uniform at $40 \mathrm{~m}$. Following a parametric convergence study, the rectangular portion of the channel was meshed using regular triangular elements of uniform size $10 \mathrm{~m}$, expanding into an unstructured mesh of variable size as the channel expanded into the semi-circular oceanic basins. The flow in the channel was driven by a head difference applied to the east and west boundaries of the model, corresponding to open oceans, enforced as a time-varying elevation. The head difference was driven by the M2 and S2 tidal constituents, which give rise to the spring-neap tidal cycle and are the dominant tidal constituents at many sites currently under consideration for tidal stream turbine deployment $[13,1]$. Slip wall conditions were enforced along the horizontal land boundaries. A uniform bed friction coefficient of $C_{D}=2.5 \times 10^{-3}$ was applied, and turbulence closure was provided by the Smagorinsky model implemented within TELEMAC-2D, in which spatial homogeneity is assumed for sub-grid scale turbulence modelling.

A fence of ten $d=20 \mathrm{~m}$ diameter tidal turbine rotors, arrayed normally to the flow direction and spanning the channel width, was deployed in the centre of the tidal channel. Two spatial degrees of freedom were available for turbine deployment; the inter-turbine spacing within a cross-stream row of turbines, and the streamwise spacing of turbines deployed in multiple rows. The ratio of the rotor swept area to the crosssectional area of the flow passage surrounding an individual turbine is described by the blockage ratio, $B=\pi d^{2} / 4(s+d) h=n \pi d^{2} / 4 w_{c} h$, where $n$ is the number of turbines in a cross-stream row. The cross-stream turbine rows were represented as continuous blocks of resistance to the flow spanning the channel width normal to the flow direction; i.e., the bypass regions around individual turbines were not explicitly represented in the simulation. The bypass flow and blockage ratio cannot be correctly represented simultaneously in a 

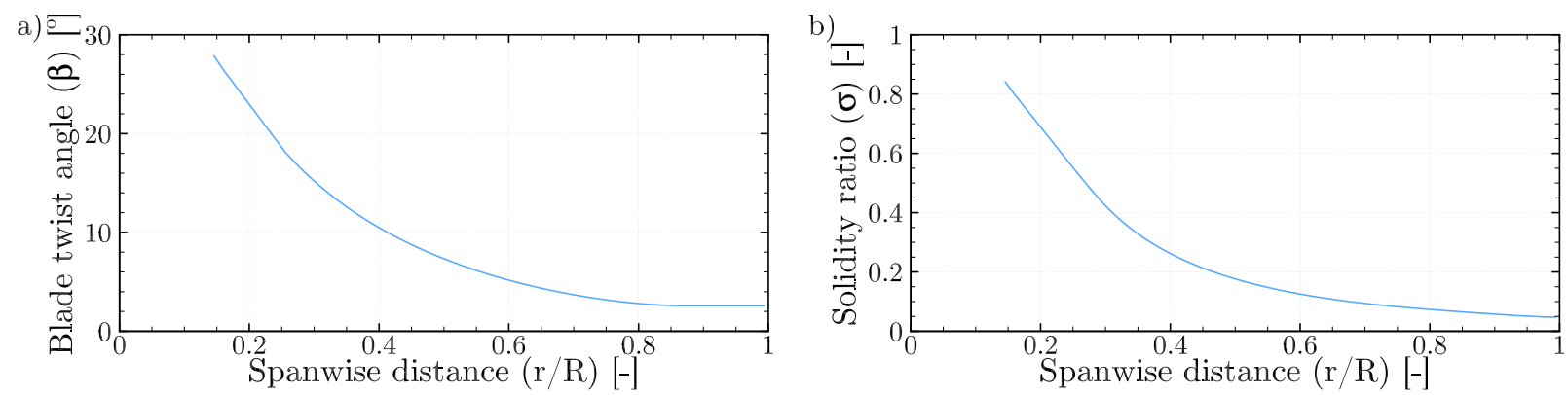

Figure 2: Spanwise variation in (a) blade twist angle $\beta$ and (b) solidity ratio $\sigma$ for the rotor designed for $B=0.196$ by Schluntz and Willden [16].

depth-averaged simulation, as information about the through depth flow variation is not preserved in the depth averaging process $[1,18]$. Instead it is necessary to employ a sub-depth scale model parameterising the relationship between rotor thrust and power in order to predict correctly the available power and also remove the correct amount of momentum from the shallow water model of the flow. The relationship between thrust, power, and velocity used herein is derived from a blockage-corrected blade element momentum model [17], eliminating the need to explicitly represent the bypass around turbines, and also avoiding the need to use correction factors for depth-averaged simulations as proposed by Kramer and Piggott [18], Vogel et al. [19]. The additional drag due to the turbine support structure is neglected, the general effect of which would be to increase the non-power producing drag in a channel, but with varying magnitude according to the support systems utilised.

\section{Rotor design and performance}

The objective of this study is to explore the impact of turbine power capping on the performance of a fence of turbines within a tidal channel. The rotor used in this study was selected to exploit the constructive interference effects that can arise when tidal turbines are closely spaced within a fence in order to boost turbine, and hence fence, power. Drawing from the wind energy industry, we examine candidate power capping strategies, pitch-to-feather and pitch-to-stall control, to better represent how turbines would operate to harness the time-varying tidal resource in the channel.

The rotor data used herein are derived from the rotor designed by Schluntz and Willden [16] for a moderate blockage configuration, $B=0.196$, shown in Figure 2. The rotor was designed using a blade element actuator disk model embedded as a set of user defined functions within ANSYS Fluent 15.0, a Reynolds-Averaged Navier-Stokes (RANS) solver. The design targeted a constant local thrust coefficient, $C_{x}=\Delta F_{x} / \frac{1}{2} \rho u_{x}^{2}$ across the rotor face, where $\Delta F_{x}$ is the applied axial force per unit area, $\rho$ is the fluid density, and $u_{x}$ is the axial flow speed through the rotor plane. The rotor blades are reasonably thick, with the Ris $\varnothing$-A1-24 section used along the entire span of the blade, and the rotor has a hub with diameter $d_{h u b}=0.15 d$. The simulated peak power coefficient $C_{P}=P / \frac{1}{2} \rho u^{3} A_{d}=0.74$, where $P$ is turbine power and $A_{d}$ is the rotor swept area, was achieved when the tip speed ratio $\lambda=\Omega d / 2 u=5$, where $\Omega$ is the rotational speed of the rotor, and $u$ is the reference flow speed. High-resolution simulations of the rotor with blade-resolved and actuator line models by Wimshurst and Willden [20] show good agreement in the thrust and power predictions with the blade element actuator disk simulations of Schluntz and Willden.

Power capping will be an important feature of tidal turbine design and operation in order to balance the capital cost of the turbine and generator with the power (and therefore revenue) available from the fluctuating tidal stream resource. An analytic BEM model accounting for the local blockage ratio is used to explore the effect of two different variable-pitch control strategies to achieve power capping: pitch-to-feather and pitchto-stall, in which it is assumed that the rotational speed of the turbine is held constant above the rated power point. These strategies represent some of the common methods used for regulating wind turbine power [21]. 
a)

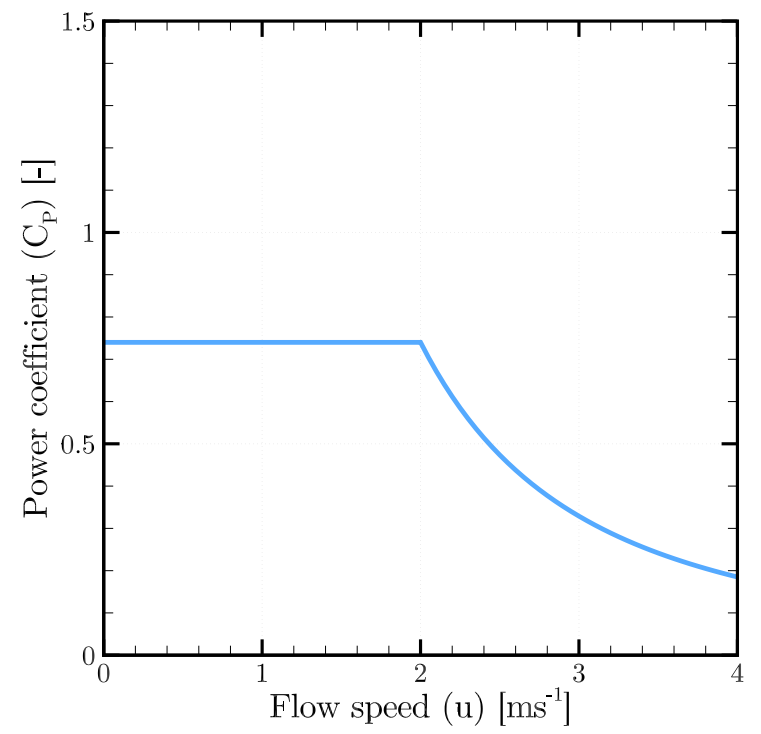

b)

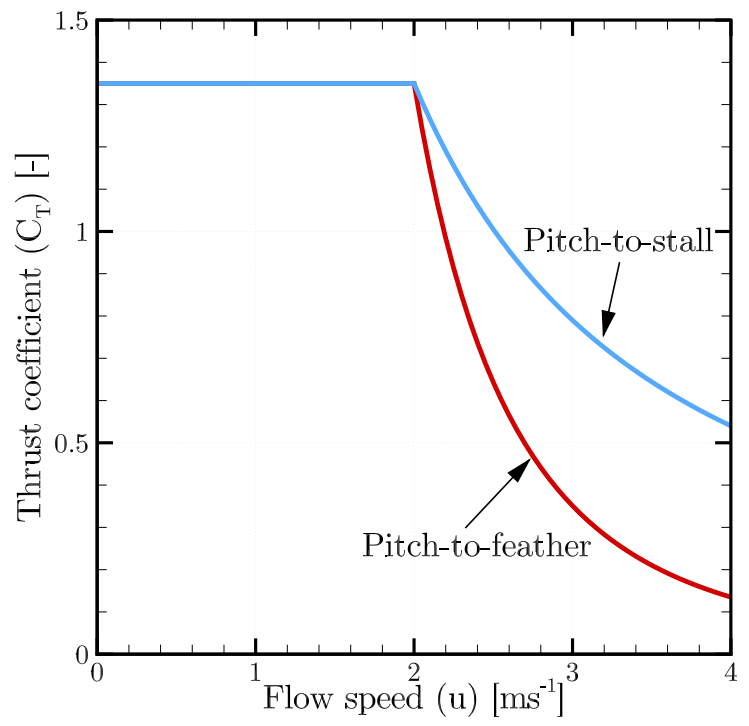

Figure 3: (a) Power and (b) thrust coefficient variation with flow speed for a single rotor with $s / d=1$ inter-turbine spacing ( $B=0.196)$. The power coefficient profile is the same for both pitch-to-feather (red) and pitch-to-stall (blue) controlled power capping, whilst the required thrust coefficient diverges above rated flow speed depending on control strategy.

The analytic model modifies blade element momentum theory for wind turbines (e.g. [21]) to account for the effects of blockage on the momentum equations in the theory following the rigid lid linear momentum actuator disk analysis of Garrett and Cummins [22]. This results in a modified relationship between the rotor induction factor and the wake induction factor depending on the blockage ratio and device thrust (c.f. the wake induction factor being twice the rotor induction factor for wind turbines), as well as rotor geometry and aerofoil lift and drag data. Tip-loss effects were accounted for using the Glauert implementation of the Prandtl tip loss model, and the analytic model solved using an iterative solution process. Further details of the blockage corrected blade element momentum model are available in Vogel et al. [17].

Until the flow reaches the rated flow speed, the turbine operates at the peak power coefficient, $C_{P}=0.74$, and corresponding tip speed ratio, $\lambda$, to maximise power. Taking the rotor design of Schluntz and Willden studied above with an inter-turbine spacing ratio of $s / d=1(B=0.196)$, the rated flow speed is $u_{r}=2.03 \mathrm{~ms}^{-1}$, yielding a rated turbine power of $1 \mathrm{MW}$. Once the rated flow speed is achieved, the power coefficient must reduce as the flow speed increases further in order to maintain the turbine's rated power until the cut-out flow speed of $u_{o}=4.00 \mathrm{~ms}^{-1}$, as shown in Figure 3. The variation of required power coefficient with flow speed is independent of whether a pitch-to-feather or a pitch-to-stall power capping control strategy is employed.

Figure 3 also shows the variation of thrust coefficient, $C_{T}=T / \frac{1}{2} \rho u^{2} A_{d}$, where $T$ is turbine thrust, with flow speed. The rotor operates at the thrust coefficient $C_{T}=1.35$ which yields the maximum power coefficient until rated flow speed is reached. The thrust coefficient reduces above rated flow speed, although the change in thrust coefficient differs between the pitch-to-feather and pitch-to-stall power capping strategies. The thrust coefficient reduces more rapidly in the pitch-to-feather case because the reduction in blade pitch angle results in a reduced angle of attack and hence smaller blade forces in both the circumferential and axial directions. The pitch-to-feather thrust coefficient reduces at a rate greater than $u^{2}$, so there is an overall reduction in rotor thrust, whereas the pitch-to-stall thrust coefficient reduces at a rate less than $u^{2}$, and consequently there is an increase in rotor thrust above rated flow speed, although the rate of increase in thrust is slower than that during pre-rated power operation. 


\section{Array Performance}

The peak spring tide flow speed in the channel across the M2+S2 tidal cycle without the presence of turbines is $3.54 \mathrm{~ms}^{-1}$, reducing to $1.78 \mathrm{~ms}^{-1}$ at the neap tide. The amplitude of the M2 tidal constituent is $0.12 \mathrm{~m}$, and $0.06 \mathrm{~m}$ for the $\mathrm{S} 2$ constituent. We start by investigating the performance of a single row of ten turbines uniformly spaced across the channel cross-section, such that $B=0.196$. The tidal array is analysed operating in three configurations: without a power capping control strategy in order to maximise instantaneous power removal from the channel; with pitch-to-feather, and pitch-to-stall power capping both with constant rotational speed above rated flow speed. The turbines are assumed to operate at the hydrodynamically optimal power coefficient below the rated flow speed, $u_{r}=2.03 \mathrm{~ms}^{-1}$ in all cases. Above the rated flow speed the non-power capping turbines continue to operate at the hydrodynamically optimal power coefficient and corresponding thrust coefficient, whereas the thrust and power coefficient change in the pitch-to-feather and pitch-to-stall case as a consequence of the changing hydrodynamic performance of the turbines as the blade root pitch angle is changed, as described in Section 2. It is assumed for simplicity that the operating conditions are uniform for all rotors in the row.

The peak power available to the array of ten turbines each tidal cycle when the channel is driven by the M2 and S2 tidal constituents is shown in Figure 4. The maximum power available to the array when power capping control is not implemented varies between a peak of $30.5 \mathrm{MW}$ at the spring tide, and $4.4 \mathrm{MW}$ at the neap tide. The mean array power over the spring-neap tidal cycle is $8.1 \mathrm{MW}$. As the magnitude of the available tidal resource is generally considerably less than $30.5 \mathrm{MW}$, it may be economically advantageous to limit the rated power of the array in order to reduce turbine costs. Setting the rated array power to be $10 \mathrm{MW}$, the maximum power available to the array is less than $10 \mathrm{MW}$ for approximately 120 hours each spring-neap tidal cycle, over which period there is no difference between the power capped and non-power capped arrays. The power available to the power capped array is virtually independent of the selection of pitch-to-feather or pitch-to-stall power capping control, as the power harnessed by the array is relatively small compared to the overall power available in the channel. Reducing the maximum (rated) power of the array by approximately two-thirds, from $30.5 \mathrm{MW}$ to $10 \mathrm{MW}$, when power capping is implemented results in a one-third reduction in the the mean array power, from 8.1 MW to $5.4 \mathrm{MW}$, over the spring-neap tidal cycle. Consequently, the array capacity factor, $C F$, defined as the ratio of the power extracted by the array to the maximum (rated, where defined) power of the array, improves from $26.6 \%$ to $54.3 \%$ when power capping is implemented as the array achieves the maximum (rated) power for a greater proportion of time, which indicates more efficient utilisation of the installed capacity of the tidal turbine array.

Figure 4 shows the variation in array thrust magnitude when peak volume flow rate is achieved in the channel over the spring-neap tidal cycle for the ten turbine arrays. The thrust always opposes the direction of the flow, and is defined to be positive in the negative $x$ direction. The maximum magnitude of array thrust varies between 18.8 MN and 5.2 MN for the non-power capping array, with a mean thrust magnitude of 6.8 MN. There is no difference in the thrust between the power capping and non-power capping cases when the channel flow speed is below the rated flow speed $u_{r}=2.03 \mathrm{~ms}^{-1}$ as there is no difference in array thrust coefficient in the three cases. Array thrust for the pitch-to-feather controlled array when the peak volume flow rate is achieved each tidal cycle is reduced when the flow speed in the channel exceeds $u_{r}$ because array thrust is reduced above rated flow speed.

The maximum thrust applied to the flow is therefore 9.0 MN, which is the array thrust when rated power is achieved. The reduction in array thrust occurs because the increase in blade pitch angle results in a reduction in the axial (thrust-producing) forces as well as the tangential (torque-producing) forces. The array thrust magnitude reduces from $9.0 \mathrm{MN}$ at rated flow speed by almost half to approximately $4.6 \mathrm{MN}$ at the maximum flow speed achieved at the spring tide in the pitch-to-feather case. This is just $38 \%$ of the corresponding thrust of $12.1 \mathrm{MN}$ for the pitch-to-stall controlled array, which occurs at the same time as the peak volume flow rate, due to the higher thrust coefficient of the pitch-to-stall controlled turbines, as shown in Figure 3. The mean array thrust magnitude over the spring-neap tidal cycle is reduced when power capping control is implemented, with a mean thrust of $4.5 \mathrm{MN}$ in the pitch-to-feather control case, and 5.8 MN in the pitchto-stall control case. The maximum reduction in thrust of the pitch-to-feather controlled array when above rated flow speed is reduced as the maximum flow speed in the channel reduces over the spring-neap tidal 

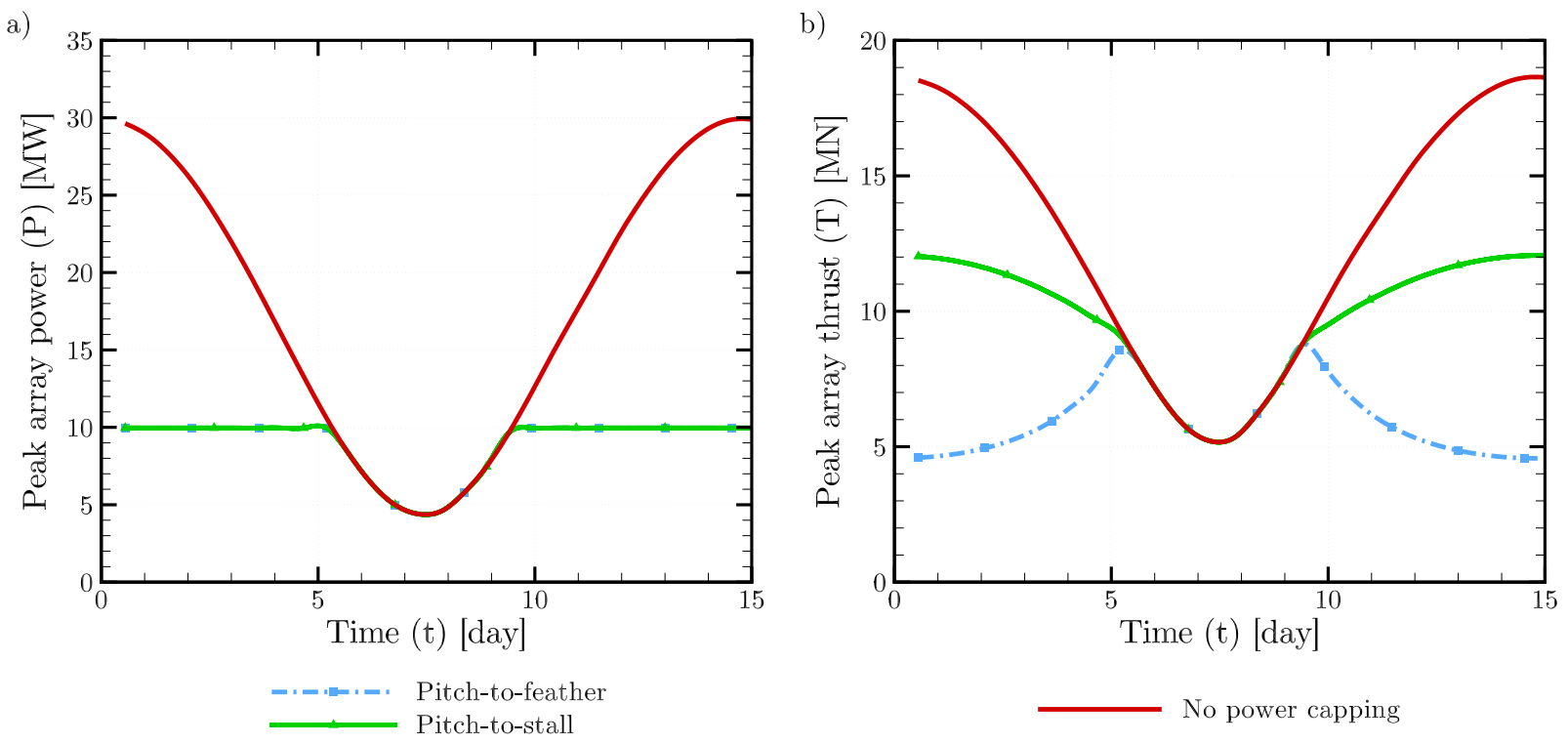

Figure 4: Peak power (a) and thrust (b) of a ten turbine tidal array in a channel driven by the M2 and S2 tidal constituents over a period of 15 days. Three arrays are simulated; an array without power capping (red), a 10 MW array with pitch-to-feather power control (dot-dash blue), and a $10 \mathrm{MW}$ array with pitch-to-stall control (dashed green). The peak power available to the pitch-to-feather and pitch-to-stall results are very similar but necessarily different due to flow rate feedback effects.

cycle, such that for cycles around neap tide there is little or no difference in power or thrust for the three arrays tested.

The effect that the different levels of thrust applied by the three arrays have on the tidal resource in the channel is illustrated in Figure 5, which shows the variation in peak volume flux through the channel over the spring-neap tidal cycle. The peak volume flux through the channel when no turbines are deployed varies between $56.7 \times 10^{3} \mathrm{~m}^{3} \mathrm{~s}^{-1}$ at the spring tide and $28.8 \times 10^{3} \mathrm{~m}^{3} \mathrm{~s}^{-1}$ at the neap tide. The volume flux through the channel is necessarily reduced from the undisturbed (natural) case when turbines are deployed due to the increased resistance to the flow presented by the turbine array. The peak volume flux is reduced by the same amount in all three cases between days 5 and 9, when the peak flow speed in the channel does not exceed the rated flow speed of the array.

At the neap tide, the volume flux through the channel is $25.0 \times 10^{3} \mathrm{~m}^{3} \mathrm{~s}^{-1}$ in the presence of the turbine array, $87.8 \%$ of that of the undisturbed channel volume flux. When the peak flow speed in the channel exceeds the rated flow speed of the array, the peak volume flux through the channel is reduced in comparison to that of the undisturbed channel by an amount that depends on the thrust imposed by the array on the flow. The peak volume flux is reduced to $83.1 \%$ of the undisturbed spring tide peak volume flux when power capping is not implemented, due to the large thrust applied to the flow. The pitch-to-stall power capping strategy reduces this thrust, resulting in an increase in peak volume flux to $88.9 \%$ of the undisturbed spring tide peak, and the pitch-to-feather control strategy reduces the thrust even further, so that the spring peak volume flux is only reduced to $95.3 \%$ of that of the undisturbed channel. Consequently, implementing power control strategies may allow some impacts of energy extraction above rated flow speed on the underlying tidal resource to be mitigated. It should be noted the effect of underwater infrastructure, such as turbine supports, not modelled here, would be to reduce the volume flow rate through the channel further. The effect of support structure resistance on flow rate, and hence power, is non-linear due to its dependency on flow speed squared and hence power capping strategy employed. 


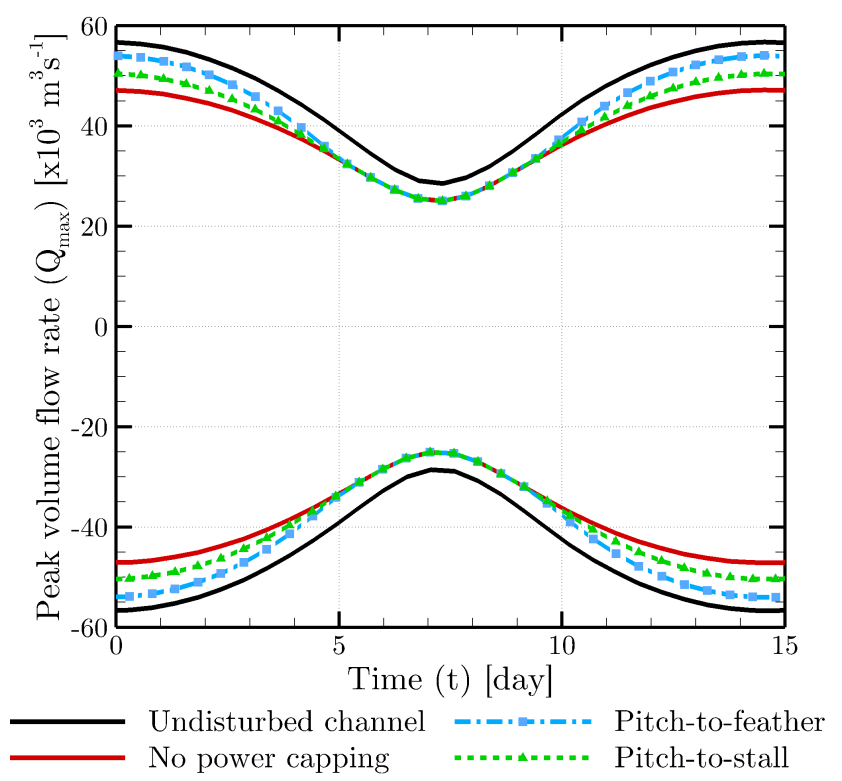

Figure 5: Peak volume flux through a tidal channel driven by the M2 and S2 tidal constituents over a period of 15 days. The peak volume flux through the channel without power extraction is shown for reference in black. Deploying an array of ten turbines results in a reduction in the peak volume flux, the magnitude of the reduction depending on whether or not power capping (red) control is used, or, if so, pitch-to-feather (dot-dash blue) or pitch-to-stall (dashed green) control strategies are employed.

\subsection{Limit of energy extraction}

The maximum power available to a farm of tidal turbines within a head-driven tidal channel is constrained by the reduction in volume flow rate that occurs as a result of the resistance that the farm presents to the flow through the channel as the number of turbines and thus installed farm power increases. Here, turbines are uniformly distributed in a farm comprised of multiple rows of turbines arrayed normally to the flow direction to investigate the maximum available power to a tidal farm within the idealised tidal channel driven by the M2 and S2 tidal constituents. The maximum number of turbines in each cross-stream row is taken to be 10, as in the previous section, such that the maximum blockage ratio of such a row of turbines is the same as in the previous section, $B=0.196$. The blockage ratio of rows with fewer than ten turbines is necessarily less than this maximum. Because each row of the array occupies the full cross-section of the channel, array-scale remixing of the wake occurs over the scale of several device diameters.

Figure 6 shows the change in farm thrust and reduction in peak volume flow rate through the channel as the number of turbines within the tidal farm increases for two power capping strategies and the no power capping base case. The maximum farm thrust over the spring-neap cycle (shown with solid lines), which occurs at the spring tide, initially increases rapidly as the number turbines in the farm increases, and then increases less rapidly once there is a sufficient number of turbines deployed in the channel, circa 45 . At this point the resistance presented by the farm is large enough so that the peak flow speed achieved in the channel is reduced below the rated flow speed for the turbines. The difference in farm thrust between the different control cases reduces to zero (although farm thrust continues to increase) once sufficient turbines are deployed within the farm because the turbines always operate at below the rated flow speed, and hence there is no difference in farm thrust and power characteristics. With fewer than 45 turbines, maximum farm thrust is greatest in the no power capping case because the turbines always operate at the hydrodynamically optimal condition to maximise power, and hence a large thrust must be applied at the spring tide. Consequently, the no power capping turbine farm results in the most significant reduction in peak volume flow rate in the channel, as the peak volume flow rate is related to the farm thrust.

The power capping strategies reduce farm thrust above rated flow speed to less than that of the non-power 

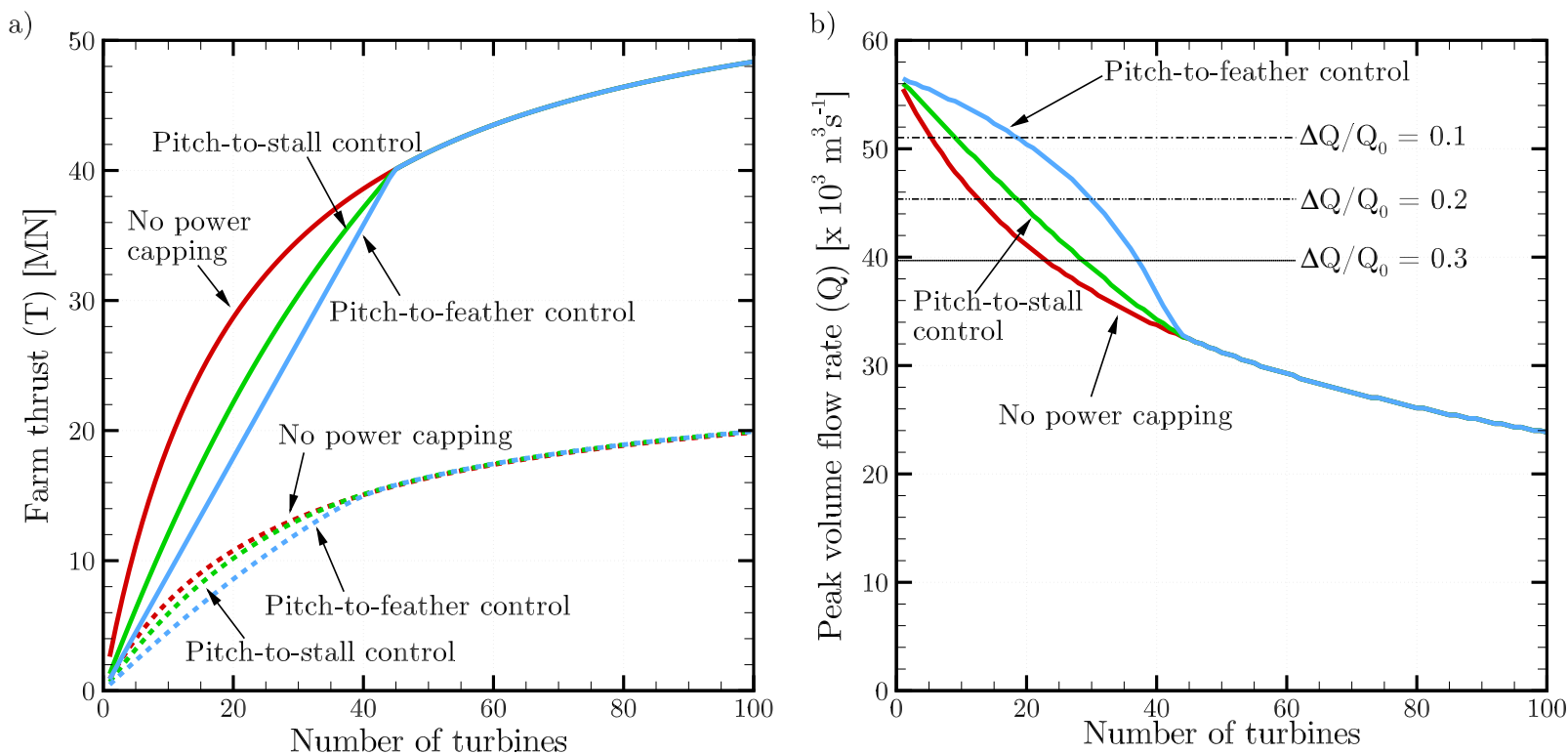

Figure 6: Farm mean (dashed lines) and peak (solid lines) thrust (a) and peak volume flow rate in the tidal channel (b) over the spring-neap tidal cycle as a function of the number of turbines in the tidal farm. Results for the case without turbine power capping are shown in red, the pitch-to-feather control strategy are shown in blue, and green for the pitch-to-stall control strategy. Three changes in peak volume flow rate from the natural peak volume flow rate $\Delta Q / Q_{0}$ are also indicated.

capping farm in order to maintain rated power, and hence there is a less significant reduction in the peak volume flow rate as compared to the non-power capping farm. In the pitch-to-feather power control case, the maximum farm thrust increases linearly with turbine number until there are 45 turbines because the maximum thrust occurs when the rated flow speed is achieved, and at higher flow speeds farm thrust reduces as the turbine blades are feathered to maintain rated power, as was seen in Figure 4. The peak volume flow rate is maintained at the highest level of the three control strategies in the pitch-to-feather case as the number of turbines increases because the farm resistance is reduced above rated flow speed. The magnitude of farm thrust reduction is greatest at the highest flow speeds, thus there is relatively little change in peak volume flow rate for small numbers of turbines. As the number of turbines increases in the pitch-to-feather controlled farm, the magnitude of farm thrust reduction above rated flow speed is reduced, and there is a greater impact on the peak volume flow rate in the channel. The total impact of the farm on volume flow rate in the channel is determined by the integral of thrust over the cycle, and can be summarised with the mean farm thrust. The difference in mean farm thrust magnitude between the different power control strategies reduces as the number of turbines increases, as compared to the maximum farm thrust, because the farms operate at the same conditions below rated flow speed, which occurs over a significant proportion of the tidal cycle.

The difference between mean thrust and maximum thrust is important in determining the lifetime structural requirements for the turbines deployed in the farm, as the devices must be designed to support the maximum forces acting on the devices. This may be significantly larger than the average magnitude of the forces acting on the devices over the spring-neap tidal cycle, as illustrated in Figure 6, particularly for relatively small numbers of turbines.

Figure 7 illustrates the variation in mean farm power over the spring-neap tidal cycle as the number of turbines within the tidal farm is increased. The non-power capping farm generally achieves the highest level of mean farm power, as the farm power is limited in both the pitch-to-feather and pitch-to-stall cases when the flow speed in the channel exceeds the rated flow speed. Mean farm power increases rapidly for small numbers of turbines, regardless of power capping strategy, although this increase is less than the rate of increase of rated farm power. Mean power per turbine, however, reduces monotonically with turbine number as the 

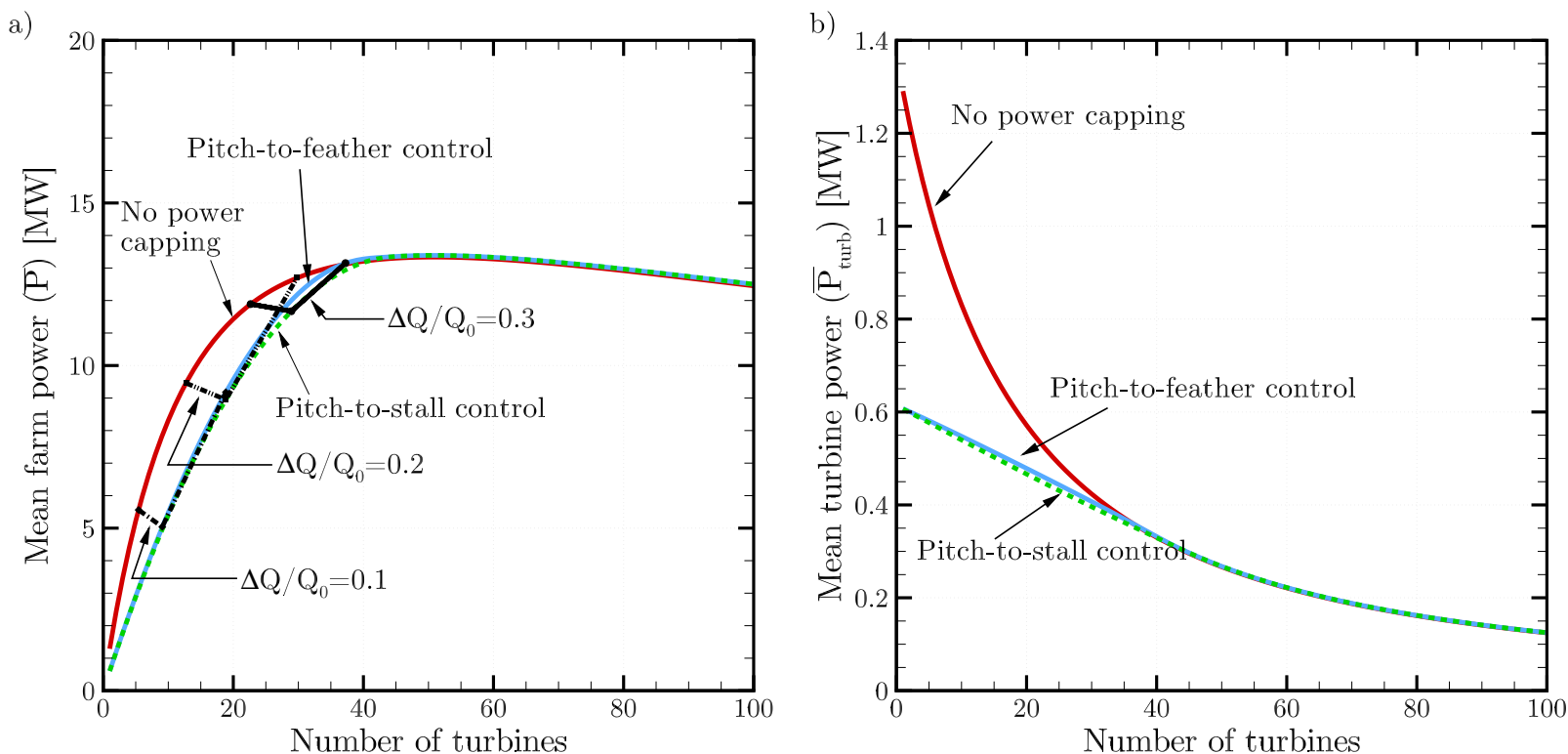

Figure 7: (a) Mean farm and (b) per turbine power averaged over the spring-neap tidal cycle as the number of turbines within the tidal farm increases. Results for the case without turbine power capping are shown in red, the pitch-to-feather control strategy are shown in blue, and green for the pitch-to-stall control strategy, with differences between the three models reducing to zero once the farm presents sufficient resistance to the flow speed that rated flow speed is never exceeded. Three changes in peak volume flow rate from the natural peak volume flow rate $\Delta Q / Q_{0}$ are also indicated.

increased flow resistance presented by the farm causes a reduction in the energy flux through the channel, limiting the power available per turbine. The rate of increase in mean farm power reduces as turbines are deployed until mean farm power is maximised, $\bar{P}=13.4 \mathrm{MW}$, with approximately 45 turbines, at which point there is also no difference between the mean farm power achieved by the different control strategies. This corresponds to the point at which the resistance presented by the farm to the flow is such that the peak flow speed in the channel no longer exceeds rated flow speed at any point during the spring-neap tidal cycle. As the number of turbines is increased above 45 the mean farm power reduces as a consequence of the very high level of resistance applied to the flow, choking the flow and reducing energy flux through the channel.

A difference in mean farm power is observed between the pitch-to-feather and pitch-to-stall controlled farms when the number of turbines is between 10 and 45, as the difference in farm thrust between the two cases is sufficient to alter the flow through the channel. The reduction in farm thrust achieved by the pitch-tofeather controlled farm above rated flow speed helps sustain a higher volume flow rate through the channel, and consequently the farm operates at rated power for a longer portion of the spring-neap tidal cycle as compared to the pitch-to-stall controlled farm, 21.6 hours vs. 17.0 hours respectively, which presents a greater resistance to the flow in the channel. The different relationship between farm thrust and power above rated flow speed, and the characteristics of the tidal channel, allows the pitch-to-feather controlled farm to achieve a very slightly higher mean farm power than with the other control strategies when the number of turbines in the farm is between $37(\bar{P}=13.1 \mathrm{MW})$ and $45(\bar{P}=13.4 \mathrm{MW})$.

The reduction in mean farm power per turbine as the number of turbines increases has an important impact on the financial viability of the farm, as the mean power of all turbines within the farm is reduced as additional turbines are added. Mean turbine power reduces significantly as more turbines are installed in the no-power capping case, whereas the mean power per turbine in the pitch-to-feather and pitch-to-stall controlled farms is less affected by the number of turbines due to their lesser impact on the flow. Consequently, there is a trade-off between the increased farm power (and thus revenue) achieved by installing additional turbines with the capital and lifetime costs of those turbines, as well as the control strategies utilised within the farms. Figure 8 illustrates the change in capacity factor as the number of turbines in the farm increases. The 


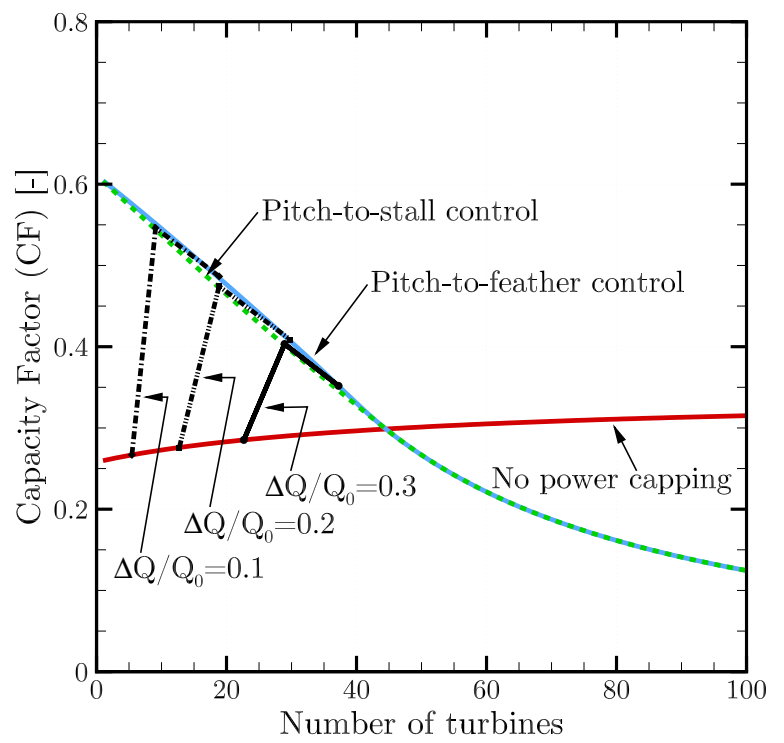

Figure 8: Change in farm capacity factor as the number of turbines in the farm increases for the pitch-to-feather (blue), pitchto-stall (dashed green) and no power capping (red) cases. Three changes in peak volume flow rate from the natural peak volume flow rate $\Delta Q / Q_{0}$ are also indicated.

capacity factor in the pitch-to-feather and pitch-to-stall cases decreases approximately linearly from $60 \%$ to $30 \%$, which is the point at which the channel flow no longer exceeds the rated flow speed. The capacity factor in the non-power capping increases slightly as additional turbines are added, from $26 \%$ for a single device to $30 \%$ when the farm consists of 45 devices, as the farm power per turbine and hence required size of turbine reduces. For this reason, a slight increase in capacity factor is observed in the no power capping case as turbines are added to farms of more than 45 devices because the maximum power per turbine continues to reduce. By comparison, the capacity factor continues to reduce for the pitch-to-feather and pitch-to-stall cases as turbine size is constant (1MW). If, for the no power capping case, capacity factor was redefined to be a fraction of the power of a single turbine at peak spring flow speed then $C F$ would be observed to decrease with turbine number also.

\subsection{Effect of flow rate reduction constraint}

If the permissible change in peak volume flow rate, $\Delta Q / Q_{0}$, is limited by environmental considerations, then the maximum number of turbines that can operate in the farm will depend on the control strategy employed. Specifying a limit of $\Delta Q / Q_{0}=0.1$, a farm without power capping would be limited to only 5 turbines, achieving a mean farm power of $5.6 \mathrm{MW}, 41.8 \%$ of the maximum power of the channel, whereas it would be possible to deploy 9 turbines with pitch-to-stall power control to extract $5.0 \mathrm{MW}, 37.3 \%$ of the channel maximum, and 19 turbines with pitch-to-feather control to extract $9.2 \mathrm{MW}, 68.7 \%$ of the channel maximum. The capacity factor of the non-power capping farm would be $26.7 \%$, whereas the pitch-to-stall and pitch-to-feather controlled farms would achieve capacity factors of $54.6 \%$ and $48.7 \%$ respectively. The capacity factor is lower in the pitch-to-feather case than for pitch-to-stall because the increased number of turbines deployed in the pitch-to-feather controlled farm is not matched by the same scale of increase in mean farm power between the two cases.

Increasing the maximum permissible change in peak volume flow rate further to $\Delta Q / Q_{0}=0.2$ and 0.3 allows more turbines to be deployed in the channel and greater farm power in all cases, although the increase in mean farm power is subject to diminishing returns as further turbines are deployed. For all specified volume flow rate reductions pitch-to-stall control always gives the lowest farm power (up to $0.5 \mathrm{MW}$ less than the no power 
capping farm), but yields the highest capacity factor due to the smaller number of turbines as compared to the pitch-to-feather case. The pitch-to-feather controlled farm, when $\Delta Q / Q_{0}=0.3$, achieves a mean farm power of $\bar{P}=13.1 \mathrm{MW}$ with 37 turbines, $98 \%$ of the maximum power available from the channel (achieved at 45 turbines), although the capacity factor is reduced to $35.2 \%$. This is higher than the non-power capping farm for the same peak volume flow rate restriction, which has a capacity factor of $28.5 \%$, but significantly below the $40.3 \%$ capacity factor of the pitch-to-stall controlled farm. The lower farm thrust that is achieved with pitch-to-feather control allows the farm to more efficiently utilise the permitted tidal resource as compared to the pitch-to-stall and non-power capping cases, both of which would require approximately 5 more turbines than the pitch-to-feather controlled farm in order to achieve a mean farm power of $\bar{P}=13.1 \mathrm{MW}$, at the environmental cost of further reducing the peak volume flow rate to $\Delta Q / Q_{0} \approx 0.4$.

\subsection{Farm design implications}

Although the precise impact of energy extraction on a tidal channel is specific to the combination of tidal channel dynamics and the farm and turbine configuration, a number of generalised implications for the design of tidal farms may be drawn. Analysing farm performance of tidal rotors, rather than actuator disks, reveals that there is a limit of the number of turbines that can be practically, or indeed would be desirable to be deployed. Specifically, the reduction in flow rate through the channel as the aggregate resistance presented by the farm increases with additional turbines means that there is a diminishing marginal benefit to farm power with each new turbine, as shown in Section 3.1. The rate at which this occurs will be dependent on the underlying dynamics of the channel. Large channels with high background friction will be relatively insensitive to the increase in resistance with each additional turbine, whereas the flow in small channels with low friction will be significantly affected by additional turbines.

Turbine power capping sacrifices some of the power available in the channel to improve the economics of the tidal farm by better matching the capital expenditure required for the turbines to the lifetime revenue available from the farm power. For the example presented herein, pitch-to-feather control was found to be the best power capping strategy. In general, it is desirable for the power capping strategy to result in reduced thrust above rated flow speed, as this reduces the resistance presented by the farm and helps to sustain the inertia of the flow through the channel, similar to that discussed by Vennell and Adcock [9]. The choice of control strategy, and its impact on farm thrust, is particularly important when constraints on the permitted change in peak volume flow rate are enforced, as might be required for environmental impact concerns. Reducing farm thrust through the power capping strategy was shown to allow more turbines to be installed, and hence a greater farm power achieved, for a given reduction in volume flow rate. It should be noted that it may be necessary to actively control the turbines below rated flow speed (particularly around neap tides) to limit the effect on the volume flow rate, depending on the rated farm power and undisturbed tidal resource at the neap tide, as was illustrated in Figure 5.

Understanding the interaction between farm operation and the tidal resource is important for determining the turbine operating conditions. Power control strategies that result in a reduction in thrust mean that the maximum (quasi-steady) loading condition for the turbine blades occurs at the rated flow speed, whereas this condition would otherwise occur at the maximum flow speed in the channel. In all cases, the reduction in flow speed through the channel due to turbine operation means that a conservative design condition would be based on the peak undisturbed channel flow speed. The study furthermore highlights the potential benefit of power capping strategies, and the resultant reduction in farm thrust, on the economics of farm design, as reflected by the capacity factor. As discussed above, the capacity factor is dependent upon the interaction between the channel dynamics and the device and farm characteristics. However, the general trend of a decreasing capacity factor as additional turbines are installed follows from the increasing farm resistance and hence reducing tidal energy resource in the channel.

\section{Conclusions}

Turbine thrust and power in tidal channels is a function of the tidally-varying channel flow speed, and maximising turbine performance is a trade-off between a range of turbine and site-specific parameters. Power 
capping offers a means to better match the revenue available from a fluctuating tidal resource with the cost (and performance) of tidal stream turbines. Simulations of an array of ten turbines in an idealised tidal channel show that array thrust reduced in rated power operation for both pitch-to-feather and pitch-to-stall power capping strategies, as compared to the non-power capping case. Pitch-to-feather control has been successfully used in the wind energy industry, whereas analysis of idealised tidal turbines (e.g. [9] and [10]) have shown that it may be advantageous for maximising mean power to operate at high thrust levels for portions of the tidal cycle, in which case pitch-to-stall control may be preferred. Introducing power capping allowed a higher flow speed to be sustained through the array and therefore allowed rated power operation to be sustained for a longer period than would be expected from the non-power capping case. Employing power capping increased the array capacity factor from 26.6\% when power capping was not employed to $54.3 \%$ when it was, indicating that the array was better matched to the spring-neap variation in available power when power capping was used for the specific channel analysed. Power capping strategies also reduced the impact that the array had on the peak volume flow rate in the channel as turbine thrust was reduced at some points throughout the tidal cycle. At the neap tide, the peak volume flow rate was reduced to $87.8 \%$ of that in the undisturbed tidal channel at the neap tide for all control strategies as the rated flow speed was not exceeded. Without power capping, the peak volume flux was reduced to $83.1 \%$ of the undisturbed case at the spring tide, which increased to $88.9 \%$ when pitch-to-stall power control was used, and $95.3 \%$ when pitch-to-feather control was used.

Power capping offers a means to better match the revenue available from a fluctuating tidal resource with the cost of tidal stream turbines. Simulations of an array of ten turbines in an idealised tidal channel show that array thrust reduced in rated power operation for both pitch-to-feather and pitch-to-stall power capping strategies, as compared to the non-power capping case. This allowed a higher flow speed to be sustained through the array and therefore allowed rated power operation to be sustained for a longer period than would be expected from the non-power capping case. Employing power capping increased the array capacity factor from $26.6 \%$ when power capping was not employed to $54.3 \%$ when it was, indicating that the array was better matched to the spring-neap variation in available power when power capping was used. Power capping strategies also reduced the impact that the array had on the peak volume flow rate in the channel. At the neap tide, the peak volume flow rate was reduced to $87.8 \%$ of that in the undisturbed tidal channel at the neap tide, for all control strategies as the rated flow speed was not exceeded. Without power capping, the peak volume flux was reduced to $83.1 \%$ of the undisturbed case at the spring tide, which increased to $88.9 \%$ when pitch-to-stall power control was used, and 95.3\% when pitch-to-feather control was used.

As the number of turbines in the tidal farm was increased, the peak flow rate in the channel reduced more slowly in the pitch-to-feather case in comparison to the pitch-to-stall and non-power capping cases because the reduction in thrust above rated flow speed helped sustain higher flow rates through the channel. Consequently pitch-to-feather power capping achieved a higher mean farm power than pitch-to-stall power control. For a farm of between 37 and 45 turbines, pitch-to-feather power control achieved the highest mean farm power of the three power control strategies investigated.

The overall extractable energy resource is constrained by a flow choking effect that arises when too large a resistance is presented to the flow. This choking effect occurred when 45 turbines were deployed in the idealised channel considered herein, resulting in a maximum mean farm power of $13.4 \mathrm{MW}$, and corresponds to the point at which the peak flow speed in the channel over the spring-neap tidal cycle is the rated flow speed of the tidal turbines. There was no difference in farm power between the three different power control strategies for farms with more than 45 turbines, as the flow rate in the channel did not exceed the turbine rated flow speed, and hence there was no hydrodynamic difference in the operational characteristics of the farms.

If the maximum change in volume flow rate in the channel is limited, the efficiency with which tidal resource is harnessed by farms with the different power capping strategies results in significant differences in permissible farm size. Limiting the change in peak volume flow rate to $10 \%$ of that in the undisturbed channel, a pitch-to-feather controlled farm would consist of 19 turbines, and achieve a time-mean power of $68.7 \%$ of the maximum channel power, as compared to a farm of 9 pitch-to-stall controlled turbines achieving $37.3 \%$ of the maximum time-mean channel power, and $41.8 \%$ for a non-power capping controlled farm of 5 turbines. 
The difference between control strategies is more significant with lower permitted changes in the volume flow rate in the channel.

\section{Acknowledgements}

The authors wish to acknowledge the support of the Oxford Martin School.

\section{References}

[1] Vennell R, Funke SW, Draper S, Stevens C, Divett T. Designing large arrays of tidal turbines: A synthesis and review. Renewable and Sustainable Energy Reviews 2015;41:454-72.

[2] Plumb RA, Marshall J. Atmosphere, Ocean, and Climate Dynamics. New York: Academic Press; 2007.

[3] Pugh DT. Tides, surges, and mean sea level. Chichester: John Wiley \& Sons; 1987.

[4] Black, Veatch . Phase II: UK tidal stream energy resource assessment. Tech. Rep.; Carbon Trust Marine Energy Challenge; 2005.

[5] Garrett C, Cummins P. The power potential of tidal currents in channels. Proceedings of the Royal Society: A 2005;461:2563-72.

[6] Vennell R. Tuning turbines in a tidal channel. Journal of Fluid Mechanics 2010;663:253-67.

[7] Draper S, Adcock TAA, Borthwick AGL, Houlsby GT. An electrical analogy for the pentland firth tidal stream power resource. Proceedings of the Royal Society: A 2013;470:20130207.

[8] Draper S, Houlsby GT, Oldfield MLG, Borthwick AGL. Modelling tidal energy extraction in a depthaveraged coastal domain. IET Renewable Power Generation 2010;4:545-54.

[9] Vennell R, Adcock TAA. Energy storage inherent in large tidal farms. Proceedings of the Royal Society: A 2014;470:20130580.

[10] Vennell R. An optimal tuning strategy for tidal turbines. Proceedings of the Royal Society: A 2016;472:20160047.

[11] Serhadglıŏlu S, Adcock TAA, Houlsby GT, Draper S, Borthwick AGL. Tidal stream energy resource assessment of the Angelsey Skerries. International Journal of Marine Energy 2013;3-4:98-111.

[12] Walters RA, Tarbotton MR, Hiles CE. Estimation of tidal power potential. Renewable Energy $2013 ; 51: 255-62$.

[13] Adcock TAA, Draper S, Houlsby GT, Borthwick AGL, Serhadglıŏlu S. Tidal stream power in the Pentland Firth - long term variability, multiple constituents, and capacity factor. Proceedings of the Institute of Mechanical Engineers Part A: Journal of Power and Energy 2014;228(8):854-61.

[14] Divett T, Vennell R, Stevens C. Channel-scale optimisation and tuning of large tidal turbine arrays using LES with adaptive mesh. Renewable Energy 2016;86:1394-405.

[15] Masters I, Chapman JC, Willis MR, Orme JAC. A robust blade element momentum theory model for tidal stream turbines including tip and hub loss corrections. Journal of Marine Engineering and Technology 2011;10(1):25-35.

[16] Schluntz J, Willden RHJ. The effect of blockage on tidal turbine rotor design and performance. Renewable Energy 2015;81:432-41. 
[17] Vogel CR, Willden RHJ, Houlsby GT. Blade element momentum theory for a tidal turbine. Submitted to Renewable Energy 2016;.

[18] Kramer SC, Piggott MD. A correction to the enhanced bottom drag parameterisation of tidal turbines. Renewable Energy 2016;92:385-96.

[19] Vogel CR, Willden RHJ, Houlsby GT. Power available from a depth-averaged simulation of a tidal turbine array. Renewable Energy 2017;114, B:513-24.

[20] Wimshurst A, Willden RHJ. Computational analysis of blockage designed tidal turbine rotors. In: Second International Conference on Renewable Energies Offshore. Lisbon, Portugal; 2016,.

[21] Burton T, Sharpe D, Jenkins N, Bossanyi E. Wind Energy Handbook. West Sussex: John Wiley \& Sons, Ltd.; 2001.

[22] Garrett C, Cummins P. The efficiency of a turbine in a tidal channel. Journal of Fluid Mechanics 2007;588:243-51. 